\title{
APPLICATIONS OF OPERATIONS RESEARCH IN FORMULA ONE
}

\author{
Aditi Mehta, Aashima Lakra, Adnan Godil, Aastik Dudani, Amaan Shaikh \\ Affiliation: Narsee Monjee Institute of Management Studies, Mumbai \\ Students, Department of Finance, Anil Surendra Modi School of Commerce, Mumbai, India
}

\begin{abstract}
Formula one is one of the world's most high-paced sports in which a fraction of seconds can cost a win. From the method of calculating the time spent on a pitstop, when the car should come for the pitstop, fuel consumption, race strategy, aerodynamics, mechanics, engine power is all influenced by operation research. The paper aims at different techniques such as optimization and simulation, further discussing the different types of methods used. It provides brief information about the outcomes, drawbacks, and recommendations given for the efficient use of these techniques in the $F 1$ racing world. It also addresses improvements that can be done by logistics, the necessary factors which need to be considered in the simulation model, and so on. The main objective is to study these techniques and provide an overview and limitations of each method, emphasizing the need for additional research to find solutions.
\end{abstract}

Keywords- Optimization, simulation, formula one, pitstops, aerodynamics, Drag force, downforce, Monte Carlo, discrete event simulation, transportation problem, race strategy, operation research

\section{INTRODUCTION}

The goal of a Formula 1 competition is to determine the winner of a race. The driver who crosses the finish line first after completing a predetermined number of laps is declared the winner.

The Formula One World Championship season is a series of Formula One races held over a certain period of time, generally a year. Each race as well as the season is referred to as a 'Grand Prix'. As of 2021, ten teams with two cars each are allowed to compete in the Formula One World Champion-ship. However, the FIA regulations limit the championship to 26 vehicles. The top ten drivers, at the end of each Grand Prix, will be awarded points based on their finishing positions. The results of all Grand Prix races held during a season are combined to determine two annual Championship awards. They are as follows:

- Drivers' Championship Award (for the drivers)
- Constructors' Championship Award (for the constructors)

Talking about some car technicalities and the race, all F1 cars can accelerate from 0 to $100 \mathrm{mph}$ (160 $\mathrm{kmph}$ ) and decelerate back to 0 in under 5 seconds. F1 cars have reached top speeds of about $300 \mathrm{mph}$ or $185 \mathrm{mph}$ on average. Each car must guarantee that its tank is fully loaded before the start of the race. (Defector 2021).

Formula One engineering is a never-ending balancing act of compromises and optimization. Downforce-generating modifications are beneficial; however, it frequently results in a drag, which is undesirable. Oversteer is caused by too much frontal downforce, while understeer is caused by too much rear downforce. Thousands of holes are punched into each brake disc to increase surface area and hence cooling. But the same holes also give the discs a higher thermal mass, making it simpler for them to become hotter. The air traveling through the brakes cools them. On the other hand, the hot air then flows through the wheels and tires, potentially overheating them. (The complete guide to Understanding Formula 1. (Defector 2021).

Each season, teams create a brand-new car and devote enormous resources to improving it. Because of the rapid rate of upgradation, new rules are frequently enacted. Such as this year's compulsory adjustments to the design of the car's floor, to intentionally slow the cars down (the current crop of cars was generating more downforce than the tires could handle). DRS allows an automobile to open a letterbox-shaped gap in its back wing. Thus, allowing air to pass through and lowering drag which enables easier overtaking. (Tippett, B. T. 2021)

Formula 1 cars are at the highest level of innovation and modern-day engineering design. F1 is considered one of the most technologically advanced and the most complicated categories of modern-day racing. To simplify the complexities of the sport, many OR techniques are applied. The focus of the study conducted by Bin-Ali and Smew (2021) is to optimize the front wing of the Formula 1 car to reduce lap times, get better handling and reduce the drag force while increasing the downforce. The front wing is one of the most influential parts of the car and needs to be optimized to produce a championship-winning race car. 


\section{International Journal of Engineering Applied Sciences and Technology, 2021 \\ Vol. 6, Issue 5, ISSN No. 2455-2143, Pages 160-165 \\ Published Online September 2021 in IJEAST (http://www.ijeast.com)}

The method used is the Optimization and Mathematical Modelling Method and the optimizer used is the Maple optimizer. Maple optimizer is a powerful mathematical tool that uses the global optimization technique. The next application of OR in F1 comes in logistics which uses techniques like strategy integration and interface management. Logistic operations are considered successful if the shipment reaches the right place, with the right packaging and labeling, in the right quantity, and at the specified time. Various techniques are used to optimize the process by using strategy integration and formula 1 interface management and by automated IT systems which help in giving the most optimum route and mode of transport by respecting the time and cost constraints. (Hung, N., 2020.)

The Monte Carlo simulation method is used to find every possible outcome in a race to develop a robust race strategy that takes into consideration all probabilistic events. The crucial goal in racing is to find the ranking among the participants. Only by contrasting different race simulations and the influence of different race strategies can we come up with the most effective strategy. (Heilmeier et al., 2020)

\section{LITERATURE REVIEW}

Talking about racing strategy, "Good pit stops are hard to come by when they do, they win you races"Niki Lauda, three-time F1 champion.

An operations-research paper by Bekker and Lotz (2009) simulates race strategy that plays an important role in winning the race, which emphasizes modeling the dynamics of the race to aid the evaluation as well as planning of the race strategies. Shannon's simulation world view (1975, p. 109) was used as a modeling paradigm here. In modeling this strategy, the racing cars were the entities having attributes, for example, the amount of fuel onboard. These entities then interact with activities, such as the pitstop or trying to pass a maneuver under certain conditions.

The drawback experienced in this model was that technical data for F1 cars are closely kept secrets to maintain competitive advantage. The discrete event simulation model used to simulate the $\mathrm{F} 1$ race was used in this paper by Bekker and Lotz (2009) as it allowed the time adjustment approach. Thus, it permits the racing events to be modeled on an aggregate basis which avoids the need to model each race. This also led to an advantage as it only required the input data available to the public.

Another published work, Heilmeier et al. (2020) talked about how the loss in time due to pit stop is traded off with the gain in time due to new tires. The work is based on the Monte Car-lo simulation (MCS) concept. This concept was applied by the implementation of realistic models for the probabilistic effects and then simulating a huge number of races which helped to determine the estimated distribution of the rank positions.

The paper focused on how in-circuit racing, the results de-pend not only on the driver and car performance but also on race strategy, and pit stops constitute an important part of race strategy. Since pit stops take time, one must find an effective compromise between benefit and expense. Race times are the central element of circuit racing. A good compromise be-tween accurate results, computational efforts, and parameterization effort is provided through lap-wise discretization.

In contrast, a position change from an empirical distribution function is drawn in the paper to identify the number of positions lost or won by each driver (Sulsters, 2018). To compute the difference in cumulative lap time between each driver in the circuit the overtaking model is used.

The overtaking model is important as it determines whether any driver was able to overtake another driver.

Further application of operation research was found for Optimizing the location of the pit crew and their equipment leading to ergonomic behavior while changing tires or refueling. Taking into consideration the curves, a best possible path is found. A good program that shall optimize everything for a single race can give the driver an advantage as to when it would be best to overtake, take planned stops, and whatnot.

Each F1 team has to decide how much fuel their car requires before each race and how long they will stop for refueling and changing tires, this is called race strategy in this sport. A wise decision can help a driver get more points and perhaps even a world championship. (Raeng.org.uk. 2021)

The paper by Heilmeier, A., Thomaser, A., Graf, M., \& Betz, J. (2020) offers a methodology to automate race strategy decisions. The focus is on Formula 1 racing series, thereby ensuring that the stands are optimized to replace worn-out tires. A quadratic optimization problem was set up that minimizes the race time, assuming that a race without opponents is possible to determine the initial estimate of the optimal race strategy. (Heilmeier, A., Thomaser, A., Graf, M., \& Betz, J. 2020).

Formula 1, known as the "Queen of Motorsports," is one of the events where the right resource's availability at the right time and the right location for the race, as well as logistics activities, have always been critical. The backbone of every event is logistics operations. Every race weekend consists of 3 competitive days, 10 teams consisting of 20 drivers and support crews are required to make the special "racing town," arrangements, lounges, garages, and service ready and operational no later than the Thursday before the event. There are approximately 2000 tons, divided by road, air, and 


\section{International Journal of Engineering Applied Sciences and Technology, 2021 \\ Vol. 6, Issue 5, ISSN No. 2455-2143, Pages 160-165 \\ Published Online September 2021 in IJEAST (http://www.ijeast.com)}

shipping, of the "standard requirements" of cargo after each race. Wieczorek (2021) talks about how this requires optimal solutions and transport problems to be resolved through operational research

The operation research is also needed in aerodynamics. To build the fastest cars humanity has ever seen. To achieve this, Amirah Abdul-Rahman, Fatemah Al-Failkawi (2020) talks about how the cars need to be optimized perfectly, following the regulations laid down by the governing body i.e. The FIA. The two main forces that the cars are subjected to are drag force and downforce. Drag force reduces the speed of the car, while the downforce provides grip and steady handling. The authors have tried to find the balance between these force-es to optimize the front wing of the car and to find the corresponding angle of attack and lift to drag ratios. (Bin-Ali and Smew, 2021)

Hence, we can conclude that F1 has enormous scope for the techniques of operation research in every department or job. Therefore, the following objectives will guide the study: -

1. To discuss the uncertainty in the various aspects of the racing systems using Monte Carlo simulation.

2. The validity of the SC model and the use of Monte Carlo simulation to assess the probabilistic effects for a strategy engineer for race strategies to be determined.

3. To discuss the drawbacks of discrete-event simulation and compare simulated models to actual race results

4. To optimize the front wing of the car which helps the car as a whole to get faster laps and improved control over-turns

5. To discuss optimal solutions and transport problems for the arrangements of the race by logistics

\section{METHODOLOGY}

The data used for this research paper was secondary data, mainly taken from existing research papers, reports, and articles. The data taken was first analyzed to find the commonly used and most credible techniques of Operation research in formula one. This helped us study the applications of OR and its efficiency in real-time events. Most of the data taken and the conclusions drawn are based on mathematical proof pro-vided by other researchers and articles which increased the credibility of the paper, and are further discussed in the pa-per. Some of the techniques ascertained in this paper are the Monte Carlo Simulation, Discrete Events Simulation, Optimization technique, etc. We have divided the paper in two parts mainly simulation and optimization to give a very clear picture and distinction between the two methods used. As race strategy is an integral part of formula one, we started with the simulation techniques and its application.

\section{ANALYSIS}

\section{a. SIMULATION}

\section{DISCRETE EVENT SIMULATION}

Discrete event simulation is the simulation technique used to make the race strategy. It's done with an approach based on time. This model is used for the simulation according to Shannon. The entities (cars) interact with the activities (which include making pitstops, need to refuel, overtaking). This model is estimated by combining historical and statistical data. The average car standings obtained from the simulation model of 50 independent replications as prescribed by Law and Kelton (2000, pp 513-514) were removed. Using face validation (Banks, 1998), it was found that no significant variations existed between real-world observations and were re-ported by the simulation model for the comparison of the actual and simulated values. This is one of the most commonly used models. The informal coefficient of the Spearman ranking correlation (Walpole and Myers, 1993) is used for the comparison of car positions at the end of each race with over 75 VV\&T techniques (Banks, 1998, P354). But while analyzing the assumptions of this model, there are many aspects to the real race which, under certain conditions, might not make the simulation model applicable. Some of them include: 1. The input data due to lack of availability of data on public domain and taking historical data which might not be available for the circuits recently added in the calendar, the model prepared may not take a lot of aspects into consideration.

2. Only one car can be overtaken at a time that may cause a certain disruption in the model. It was found that many estimates show that a different pit stop strategy would also have been applied by the specific team for a better position, but there's not enough evidence to prove that the actual race times were different from the race times reported in the simulation model.

3. Certain aspects of the car set-up, such as wing levels, are more easily gathered through simulations while others are harder because they depend on more factors - some not within control, such as weather.

\section{MONTE CARLO SIMULATION METHOD}

The Monte Carlo simulation method helps to find every possible outcome in a race to develop a robust race strategy that takes into consideration all probabilistic events. The crucial goal in racing is to find the ranking among the participants. The most significant probabilistic influences are accidents and 


\section{International Journal of Engineering Applied Sciences and Technology, 2021 \\ Vol. 6, Issue 5, ISSN No. 2455-2143, Pages 160-165 \\ Published Online September 2021 in IJEAST (http://www.ijeast.com)}

failures, Full course yellow and safety car phases, drivers' starting performance, variability in lap times, and pit stop durations. Previously, while calculating these probabilistic influences were not considered. (Heilmeier et al., 2020) The author infused these factors into his models, making the race strategies much more realistic. The authors in the paper have decided to discretize the race lapwise, which makes it very easy for the strategy engineer to compare strategies. The first simulation step is to calculate the lap times. (Phillips,2014) Taking into account two assumptions that every SC (safety car which is employed during hazardous construction in a race, effect on race result is high) phase is caused by an accident, and every VSC ( cars are made to slow down, effect on race is negligible) is caused by failure, thus making the models much simpler. It must be noted that accurate parameterization of the race simulation is of the utmost importance and a deciding factor of the result of the race. Heilmeier et al. (2020) extended existing ideas (using Bayesian inference for accidents and failures) and constructed new approaches to modeling important probabilistic effects, discussed earlier.

\section{b. OPTIMIZATION}

The data for this paper by Bin-Ali and Smew (2021) has been analyzed using the Optimization and Mathematical Modelling Method. In this optimization and mathematical modeling approach, the focus is on the system's most influential parts and the remaining parts are excluded. This approach studies the most influential variables and manipulates them to study the changes in the model. Mathematical model results in mathematical formulation for the model. Due to the symmetrical nature of the front wing, only half of it will be modeled. (Bin-Ali and Smew ,2021)

- The model is represented as a cantilever beam with a uniform exerted loading because of the support at the nose cone and the distributed force exerted on the main plane. (1).

- It also represents the bending on the different regions of the beam as shown in the equation. (2)

(1) $M(x)=E I \frac{D^{2} v}{d x^{2}}$

(2) $M=-\frac{w x^{2}}{2}+w L x-\frac{w L^{2}}{2}$

The following equations are bending equations that can be equated and combined.

The data was collected in two parts, the decision variables, and the constant inputs. The decision variables being (E) modulus of elasticity, (L) wing length, and (x) distance from wing to support. The constant inputs are (W) downforce, (I) second moment of inertia. (Bin-Ali and Smew ,2021)

\section{Maple Optimizer}

Maple optimizer is an optimization tool that is a powerful mathematical engine. It uses a technique known as the global optimization technique with a differential evolution to optimize differential equations with multiple variables. This optimization method (Bin-Ali and Smew, 2021) is an iterative process of selection between many initial solutions obtained under the feasible and constraint range. New combinations of these solutions are made until the optimal solution is found. This optimizer will yield results twice. The first optimization will be done to find the optimal wing that passes the deflection test without the cost constraint. The second optimization will be done by setting the modulus to the cheapest manufacturing material's modulus in an attempt to find the cheapest possible wing characteristics that will pass the deflection test, and also help in cost minimization.2nd order differential equation with several variables was used in the method to find the optimum bending of the front wing.

A study conducted used the developed optimization to find the optimal wing and the cheapest wing that passes the deflection test thereby validating the mathematical model.

\section{Logistics}

As the races are hosted on different continents within a month. Following every race, each team's cargos are shipped to the next race venue, comprising cars, garage kits, air channels, and hospitality kits. The support crew has to cross-check a 200-page checklist of items. There are 10 teams in f1 and 23 races take place in a season so the cargo of each team travels a minimum of 23 countries. There are three modes of transport Dębicki, T. (2021) used in F1 based on the urgency level and category of cargo. The cargo is mainly divided into critical and non-critical cargo. It requires $24-48$ hours to assemble or to dismantle a motorhome and garage.

Optimization under logistics: -

There is ' $k$ ' number of vehicles that are available for the logistics distribution center, (Wieczorek, A. 2020) vehicles load capacity is " $x$ " so the maximum load that can be carried is ' $\mathrm{k} * \mathrm{x}$ '- $\mathrm{I}$, the minimum function of distance can be used to find the displacement between the two drop off and pick up points which can be denoted by Ds and can be matched with the truck's mileage for optimum cost utilization 'Ds"-ii. The major constraint in formula 1 is time i.e. The time taken from the logistics center to each delivery point can be defined as $\mathrm{T}$ by using minimum function. It can be calculated by finding the average distance to the covered and average speed of the car-go and calculating the T. In all of 


\section{International Journal of Engineering Applied Sciences and Technology, 2021 \\ Vol. 6, Issue 5, ISSN No. 2455-2143, Pages 160-165 \\ Published Online September 2021 in IJEAST (http://www.ijeast.com)}

this calculation, all constraints are subject to changes except $\mathrm{X}$ which is the max load a container/truck can carry.

\section{V. $\quad$ FINDINGS AND CONCLUSION}

MCS overall helps us to understand how our strategy would work against unforeseen events. During a real race, the basic strategy must be assumed to the live situation. Although it causes some modeling difficulties, this model is preferred over other approaches because the fast computing times are essential for the intended application. They make the application of the Monte Carlo method possible in the first place. During a real race, the basic strategy must be assumed to the live situation. It is visible that MCS predictions are accurate when we compare actual and simulated race data. There are some drawbacks to this simulation method which are mentioned later in this paper.

Even though Formula 1 management takes responsibility for the logistics, it's the responsibility of the teams to financially manage their cargoes. To reduce the cost, the highest priority shipment will receive the most specialized service with time as a constraint and without even considering the cost while the non-critical shipment will not have time as a constraint and will be shipped with minimum cost. The above activities are managed with the fl schedule which is changed accordingly to optimize processes. In the search for competitive ad-vantages, teams in F1 increasingly rely on advanced simulation technology. The ultimate objective is therefore to develop a model that can analyze race strategies in real-time. The decision-maker(s) can then react with confidence to race incidents within seconds of their occurrence. This project combined multiple distinct software technologies to create a system that achieved its goals of using evolutionary computing to optimize the parameters of a Formula One car and obtaining real gains in lap times when compared to all other approaches examined. Due to the lack of research papers and literature available in this area, some areas couldn't be re-searched in depth.

\section{LIMITATIONS AND FUTURE RESEARCH DIRECTIONS}

1. Monte Carlo simulation: While most of the results obtained from the MCS method are reliable and accurate there are some drawbacks to this simulation method. Eg: fewer data available involving car accidents and racing making it difficult to factor in the results completely. Some situations cannot be simulated following lap-wise discretization, causing minor inaccuracies in the results.
However, this method has some major drawbacks, Com-mutationally inefficient. When you have a large number of variables bound to different constraints, it requires a lot of time and a lot of computations to approximate a solution using this method. Even in cases of poor parameters and constraints used as input into the model then it tends to yield fewer effective results. Yet the simulations of Monte Carlo cannot work their magic without solid, reliable input. (Heilmeier et al., 2020)

2. Logistics: Any foreseen circumstances i.e., backto-back weekend races or end moment change of venue can make it a challenging task for the ground team. The circuit changes every race which makes it difficult to bring standardization in the logistics process. (Hung, N., 2020.)

3. Discrete Events Simulation: A selected scenario cannot guarantee success, but the team can follow the proposed strategy in the knowledge that the best scenarios are expected. (J Bekker \& W Lotz (2009). Some factors which may seem insignificant, but have an important role like date of birth, nationality, and finishing status are also taken into account if racing in the country has psychological effects or if certain drivers are more likely to crash than others are not taken into consideration while creating this model. It is very difficult to model damages, accidents, and FCY phases in the simulation accurately. The complete car model cannot always be entirely precise, and you cannot structure and model the grip of the tar-mac. You can also never be entirely sure about how the tire will respond to it.

4. Front-wing Optimization: Limitations here are in the form of regulation changes that take place every year. The FIA changes the rules every year to make racing and car building competitive and interesting. This forces the teams to optimize the front wing again every year to make faster cars in each of those years. (Bin-Ali and Smew, 2021)

\section{RECOMMENDATIONS}

The data is recorded in an unrecognizable manner; it is believed that incorporating the specific details of a lap's performance into an evolutionary process will result in a considerably more successful algorithm. This would entail looking at the effectiveness of particular car components and specific areas of the track, then changing the settings in the same way as previously to account for the new knowledge. A speedier testing approach would be extremely beneficial, as it would reduce the time required for fitness testing as well as the overall optimization process. Using huge population numbers is currently unappealing due to the time required for testing.

As MCS method is very data-intensive, it is suggested to use the Latin hypercube sampling method in the future be-because it gives the results of Monte Carlo simulations faster. 


\section{International Journal of Engineering Applied Sciences and Technology, 2021 \\ Vol. 6, Issue 5, ISSN No. 2455-2143, Pages 160-165 \\ Published Online September 2021 in IJEAST (http://www.ijeast.com)}

To optimize transportation, the containers inside could be modified which could fit multiple car chassis and fragile items to utilize the space efficiently.

The transportation can be optimized by using minimization and maximization functions of variables like number of vehicles/containers available for transport, maximum load a truck/container can carry, delivery time frame, the distance between the destination, and the cost time efficient medium of transport

\section{REFERENCES}

[1] Simulation in F1 Racing: Saving Costs, Improving Outcomes \& Trialing Innovations | HealthySimulation.com. (2018). Retrieved 2 September 2021, from https://www.healthysimulation.com/17439/sim ulation-in-f1-racing-saving-costs-improvingoutcomes-trialing-innovations/

[2] Bin-Ali, J. and Smew, W., 2021. Formula one Front Wing Optimization and Configuration. [online] Ieomsociety.org. Available at: $<\mathrm{http}$ ://ieomsociety.org/ieom2018/papers/667.p df $>$ [Accessed 20 August 2021].

[3] Carrasco Heine, O. and Thraves, C., 2021. On the Optimization of Pit-Stop Strategies via Dynamic Programming. SSRN Electronic Journal.

[4] Nytimes.com. 2021. Computers are Formula One's wizards of winning (Published 2008). [online] Available at: <https://www.nytimes.com/2008/07/04/sports/ 04iht-SRSTRATEGY.1.14234141.html>

[5] Medium. 2021. The Logistics behind F1. [online] Available at: $<$ https://medium.com/speedbox-is-typing/thelogistics-behind-f1-7537e445de20>

[6] Building a race simulator. flmetrics. (2021). Retrieved 25 August 2021, from https://f1metrics.wordpress.com/2014/10/03/b uilding-a-race-simulator/.

[7] R. G. Ingalls, "Introduction to simulation," Proceedings of the 2011 Winter Simulation Conference (WSC), 2011, pp. 1374-1388, doi: 10.1109/WSC.2011.6147858.

[8] Building a race simulator. (2014). Retrieved 14 September 2021, from https://flmetrics.wordpress.com/2014/10/03/b uilding-a-race-simulator/

[9] Computers are Formula One's wizards of winning (Published 2008). (2008). Retrieved 14

[10] September 2021, from https://www.nytimes.com/2008/07/04/sports/0 4iht-SRSTRATEGY.1.14234141.html

[11] Optimal control of Formula One car energy recovery systems. (2021). International Journal of Control. Retrieved from https://www.tandfonline.com/doi/abs/10.1

[12] The Logistics behind F1. (2017). Retrieved 29 August 2021, from https://medium.com/speedbox-is-typing/thelogistics-behind-f1-7537e445de20

[13] The Complete Guide to Understanding Formula 1 | Defector. (2021). Retrieved 1 September 2021, from https://defector.com/thecomplete-guide-to-understanding-formula-1/

[14]Dębicki, T. (2008). Challenges for logistics in the pinnacle of motorsports - Formula 1. Archives Of Transport System Telematics, Vol. 1, iss. 1, 3-7. Retrieved from http://yadda.icm.edu.pl/baztech/element/bwme ta1.element.baztech-article-BSL7-0036-0001

[15] Planning Formula One race strategies using discrete-event simulation. (2021). Journal of The Operational Research Society. Retrieved from https://www.tandfonline.com/doi/abs/10.10

[16] Virtual Strategy Engineer: Using Artificial Neural Networks for Making Race Strategy Decisions in Circuit Motorsport - MDPI. (2021). Retrieved 15 September 2021, from https://www.readkong.com/page/virtualstrategy-engineer-using-artificial-neuralnetworks-2444414

[17] Hung, N. (2020). Development of the project management for organizing the logistics services to a Formula One race: A case study of a local logistics agent for Formula One Vietnam 2020 that was postponed. Retrieved 15 September 2021, from https://www.theseus.fi/handle/10024/3400

[18] Wieczorek, A. (2019). Logistics in Racing Sports on the Example of F1. Transport Economics and Logistics, 82, 143-150. doi:10.26881/etil.20 